\title{
Evaluating lower limb tendinopathy with Victorian Institute of Sport Assessment (VISA) questionnaires: a systematic review shows very-low-quality evidence for their content and structural validity-part I
}

\author{
Vasileios Korakakis $^{1,2}$ (D) Argyro Kotsifaki ${ }^{1}$ (D) Manos Stefanakis ${ }^{3}$ (D) Yiannis Sotiralis $^{2}$ (D) Rod Whiteley $^{1}$ (D) \\ Kristian Thorborg ${ }^{4}$ (D)
}

Received: 6 January 2021 / Accepted: 26 April 2021 / Published online: 21 May 2021

(c) The Author(s) 2021

\begin{abstract}
Purpose The Victorian Institute of Sport Assessment (Achilles tendon-VISA-A, greater trochanteric pain syndromeVISA-G, proximal hamstring tendinopathy_VISA-H, patellar tendon-VISA-P) questionnaires are widely used in research and clinical practice; however, no systematic reviews have formally evaluated their content, structural, and cross-cultural validity evidence. The measurement properties referring to content, structural and cross-cultural validity of the VISA questionnaires were appraised and synthesized.

Methods The systematic review was conducted according to Consensus-based Standards for the Selection of Health Measurement Instruments (COSMIN) methodology. PubMed, Cochrane, CINAHL, EMBASE, Web of Science, SportsDiscus, grey literature, and reference lists were searched. Development studies and cross-cultural adaptations (12 languages) assessing content or structural validity of the VISA questionnaires were included and two reviewers assessed their methodological quality. Evidence for content (relevance, comprehensiveness, and comprehensibility), structural, and cross-cultural validity was synthesized. A modified Grading of Recommendations Assessment Development and Evaluation (GRADE) approach was applied to evidence synthesis.

Results The VISA-A presented very-low-quality evidence of sufficient relevance, insufficient comprehensiveness, and inconsistent comprehensibility. VISA-G displayed moderate-quality evidence for sufficient comprehensibility and very-low-quality evidence of sufficient relevance and comprehensiveness. The VISA-P presented very-low-quality evidence of sufficient relevance, insufficient comprehensiveness, and inconsistent comprehensibility, while VISA-H presented very-low evidence of insufficient content validity. VISA-A displayed low-quality evidence for structural validity concerning unidimensionality and internal structure, while VISA-H presented low-quality evidence of insufficient unidimensionality. The structural validity of VISA-G and VISA-P were indeterminate and inconsistent, respectively. Internal consistency for VISA-G, VISA-H, and VISA-P was indeterminate. No studies evaluated cross-cultural validity, while measurement invariance across sexes was assessed in one study.

Conclusions Only very-low-quality evidence exists for the content and structural validity of VISA questionnaires when assessing the severity of symptoms and disability in patients with lower limb tendinopathies.

Level of evidence IV.

Registration PROSPERO reference-CRD42019126595.

Keywords Patient-reported outcome measures · Tendinopathy $\cdot$ Content validity $\cdot$ Unidimensionality $\cdot$ COSMIN

Vasileios Korakakis

Vasileios.Korakakis@aspetar.com

Extended author information available on the last page of the article

\author{
Abbreviations \\ COSMIN Consensus-based Standards for the selection \\ of health Measurement Instruments \\ PROM Patient-rated outcome measures \\ PRISMA Preferred reporting items for systematic \\ reviews and meta-analyses
}




$\begin{array}{ll}\text { VISA } & \begin{array}{l}\text { Victorian Institute of Sport Assessment } \\ \text { Victorian Institute of Sport Assessment Achil- } \\ \text { VISA-A tendinopathy }\end{array} \\ \text { VISA-G } & \begin{array}{l}\text { Victorian Institute of Sport Assessment } \\ \text { greater trochanteric pain syndrome }\end{array} \\ \text { VISA-H } & \begin{array}{l}\text { Victorian Institute of Sport Assessment proxi- } \\ \text { mal hamstring tendinopathy }\end{array} \\ \text { VISA-P } & \begin{array}{l}\text { Victorian Institute of Sport Assessment patel- } \\ \text { lar tendinopathy }\end{array}\end{array}$

\section{Introduction}

According to the International Scientific Tendinopathy Symposium Consensus from 2020, the impact of lower limb tendinopathies on the patient should be measured using validated outcome measures that can capture the core domains of the condition such as: functional testing, participation in life activities, psychological factors, physical function capacity, and most importantly disability via condition-specific patient-rated outcome measures (PROMs) [44, 78]. The Victorian Institute of Sport Assessment (VISA) questionnaires $[6,20,69,80]$ have been recommended by the 2020 consensus statement [78] and are the most used conditionspecific lower limb questionnaires in the literature $[8,11$, $37,44,52,57,76,77]$.

Four self-administered VISA questionnaires exist which assess the severity of symptoms in patients with Achilles tendinopathy (VISA-A), greater trochanteric pain syndrome (VISA-G), proximal hamstring tendinopathy (VISA-H), and patellar tendinopathy (VISA-P) $[6,20,69,80]$. Six out of eight items rate pain level during daily activities and functional tests, and two items provide information on the impact of tendinopathy in physical activity or sports participation.

The strength of a PROM can be evaluated by the COnsensus-based Standards for the selection of health Measurement INstruments (COSMIN) [64]. COSMIN evaluates validity, reliability, and responsiveness of outcome measurement instruments like the patient-reported VISA questionnaires $[6,20,69,80]$. The quality of an outcome measurement instrument is determined by its validity [55]. In turn, content validity has been suggested as the first and most important measurement property to consider when selecting a PROM $[65,74]$. Lack of content validity potentially affects all other measurement properties. For example, irrelevant items decrease internal consistency and structural validity, and missing concepts decrease validity and responsiveness [74].

Despite the widespread use of the VISA questionnaires, to our knowledge, no systematic reviews have formally evaluated their content, structural, and cross-cultural validity evidence.

The measurement properties of the VISA questionnaires were appraised and synthesized. Here, the first part of the systematic review is reported of all available VISA questionnaires for patients with Achilles tendinopathy, greater trochanteric pain syndrome, proximal hamstring tendinopathy, and patellar tendinopathy, providing researchers and clinicians with an overview appraising measurement properties concerning content, structural, and cross-cultural validity using COSMIN methodology.

\section{Materials and methods}

\section{Protocol and registration}

The search strategy and reporting of this systematic review adhered to the Preferred Reporting Items for Systematic Reviews and Meta-Analyses (PRISMA) guidelines [53], followed the COSMIN methodology for systematic review of PROMs [64], and the Cochrane group's recommendations [29]. The protocol was prospectively registered in PROSPERO (CRD42019126595).

\section{Information sources and search methods}

PubMed, Cochrane, CINAHL, EMBASE, Web of Science, and SportsDiscus databases were independently searched by two reviewers from inception of database to 19 May 2020 without language restriction, to reduce language and publication bias.

Grey literature was searched via OpenGrey.eu, and the following registries: Clinical Trials.gov and EU clinical trials register. Reference lists, citation tracking results, and systematic reviews were also manually searched.

The search strategy included a comprehensive PROM filter developed by the COSMIN group [13, 73] and two basic strings of key terms (names of instruments and population of interest) (Online Resource 1).

\section{Study selection}

Search results were imported into EndNote where duplicates were removed, and then, title and abstract were independently evaluated by two reviewers (AK and MS). Subsequently, the full text for each potentially eligible study was evaluated. Reference lists were checked for additional potentially relevant studies [64]. A third reviewer (VK) was consulted if consensus was not reached [39].

This part of the systematic review included only the eligible studies that reported on content validity, structural validity, internal consistency, and cross-cultural validity/ measurement invariance of the VISA questionnaires. The remaining measurement properties are reported and synthesized separately. 


\section{Eligibility criteria}

Content validity studies were eligible if they were full-text original articles assessing relevance, comprehensibility, or comprehensiveness of the content of the VISA questionnaires by patients or professionals. Cross-cultural adaptation studies of the questionnaires were included as content validity studies if they performed a pretest of the adapted VISA $[2,10]$. Studies evaluating the internal structure of the questionnaires (structural validity, internal consistency, and cross-cultural validity/measurement invariance) were eligible if they were full-text original articles assessing the dimensionality of the construct of the questionnaires by factor analysis, reporting on the interrelatedness among the items (Cronbach's $\alpha$ ), or evaluating if the performance of the items on a culturally adapted VISA were an adequate reflection of the performance of the items of the original version of the instrument [13, 64].

\section{Inclusion and exclusion criteria}

The general inclusion criteria were: (a) all types of studies assessing at least one measurement property of the VISA questionnaires (including development and not limited to validity, reliability, responsiveness, and interpretability), (b) including patients with greater trochanteric pain syndrome, proximal hamstring tendinopathy, patellar tendinopathy, or Achilles tendinopathy, as well as other groups of asymptomatic/injured individuals that were used in measurement properties assessment, and (c) only full-text articles in peer-reviewed journals. Following recommendations [64], studies that only used a VISA questionnaire as an outcome measurement instrument were excluded, for instance randomized-controlled trials, or studies in which a VISA was used in a validation study of another instrument.

\section{Data extraction}

Data from studies meeting the inclusion criteria were extracted independently (VK and $\mathrm{AK}$ ) using standardized extraction forms and cross-checked. Any disagreements were resolved by consensus. Publication details, sample size, patient characteristics, content validity domain evaluated and population (relevance, comprehensiveness, comprehensibility), analysis and model of structural validity assessment, and main indices and results for structural validity and internal consistency (i.e., number of factors, Cronbach's $\alpha$ ) were extracted.

\section{Assessment of the methodological quality of single studies and evaluation of results against criteria for good measurement properties}

The methodological quality of each eligible study on a measurement property was assessed separately using the
COSMIN Risk of Bias checklist [54]. The development studies and the studies on content validity were assessed using COSMIN standards; 35 items subdivided into two parts and 31 items subdivided into two parts (patients or professionals), respectively. Studies assessing internal structure were also evaluated against COSMIN standards; 4 items for structural validity, 5 items for internal consistency, and 4 items for cross-cultural validity or measurement invariance. COSMIN recommendations $[17,21]$ were used to judge important flaws in structural validity. Measurement properties from first administration of each PROM were used for evaluation where applicable.

Each standard and subsequently each study was scored on a 4-point rating scale as "very good", "adequate", "doubtful", or "inadequate" [64]. The methodological study quality score per measurement property was determined by the item with the lowest score (worse score counts) [64].

Subsequently, the results on each measurement property were rated against the updated criteria for good measurement properties [64, 72]. Content validity and internal structure were rated as "sufficient" (+), "insufficient" $(-)$, "inconsistent" $( \pm)$, or "indeterminate" (?). Additional criteria for structural validity and internal consistency good measurement properties were applied [10]. Two reviewers (AK and MS) independently rated the quality of measurement properties; in case of any rating discrepancies, consensus was resolved by discussion with a third reviewer (VK).

\section{Rating the quality of evidence}

The evidence was summarized, and the quality of evidence was judged for each measurement property separately by two independent reviewers (AK and MS) using a modified GRADE approach [64]. Evidence started at high quality and was downgraded according to the presence and extent of specific dimensions recommended for the quality of evidence in PROM measurement properties studies: risk of bias (methodological quality), inconsistency (unexplained inconsistency of results across studies), imprecision (total sample size), and indirectness (evidence from population different than that of interest). For content validity, only risk of bias, inconsistency, and indirectness are applicable [74]. The results were qualitatively summarized or quantitatively pooled (where applicable) and compared against the criteria for good measurement properties to determine whether the "overall" measurement property of the PROM is sufficient $(+)$, insufficient $(-)$, inconsistent $( \pm)$, or indeterminate (?) [64].

\section{Statistical analysis}

Pooling of internal consistency coefficients was performed using the R statistical platform [66] (metafor package) [79]. 
Initially, the coefficients were transformed [24] to stabilize the variances and approximate to the normal distribution. A random-effects model was used due to clinical and statistical heterogeneity and subgroup analyses were performed based on clinical criteria (i.e., patients or mixed sample of patients and asymptomatic individuals, age) where applicable. Values were presented as pooled mean estimate and $95 \%$ confidence intervals $(95 \% \mathrm{CI})$.

\section{Results}

\section{Study characteristics}

Of the original 1511 studies, 34 remained after duplicate removal. Of these, 31 met the eligibility criteria appraising measurement properties of: VISA-A [15, 16, 18, 28, 30, 33, 34, 41, 43, 45, 51, 69-71], VISA-G [3, 19, 20, 31], VISA-H $[6,40]$, and VISA-P [1, 7, 22, 25-27, 32, 36, 42, 46, 60, 80, $81,83]$. Twenty-four cross-cultural adaptations validated the VISA questionnaires in 12 languages. Of the eligible studies, three [19, 27, 51] did not evaluate content validity and/or internal structure of the PROMs and were excluded (Fig. 1).

\section{Quality, results, and evidence synthesis of content validity studies}

Content validity of the VISA questionnaires was evaluated by 71 patients (comprehensibility) with tendinopathy and 12 professionals (relevance). The VISA development studies were of inadequate quality $[6,20,69,80]$. The quality of the VISA content validity studies is presented in Table 1 , and the quality of evidence is presented in Table 2.

\section{VISA-A}

Very-low-quality evidence was found for inconsistent content validity of VISA-A (Table 2).

\section{VISA-G}

Very-low-quality evidence for sufficient content validity of VISA-G (Table 2).
Fig. 1 PRISMA flow diagram for study inclusion

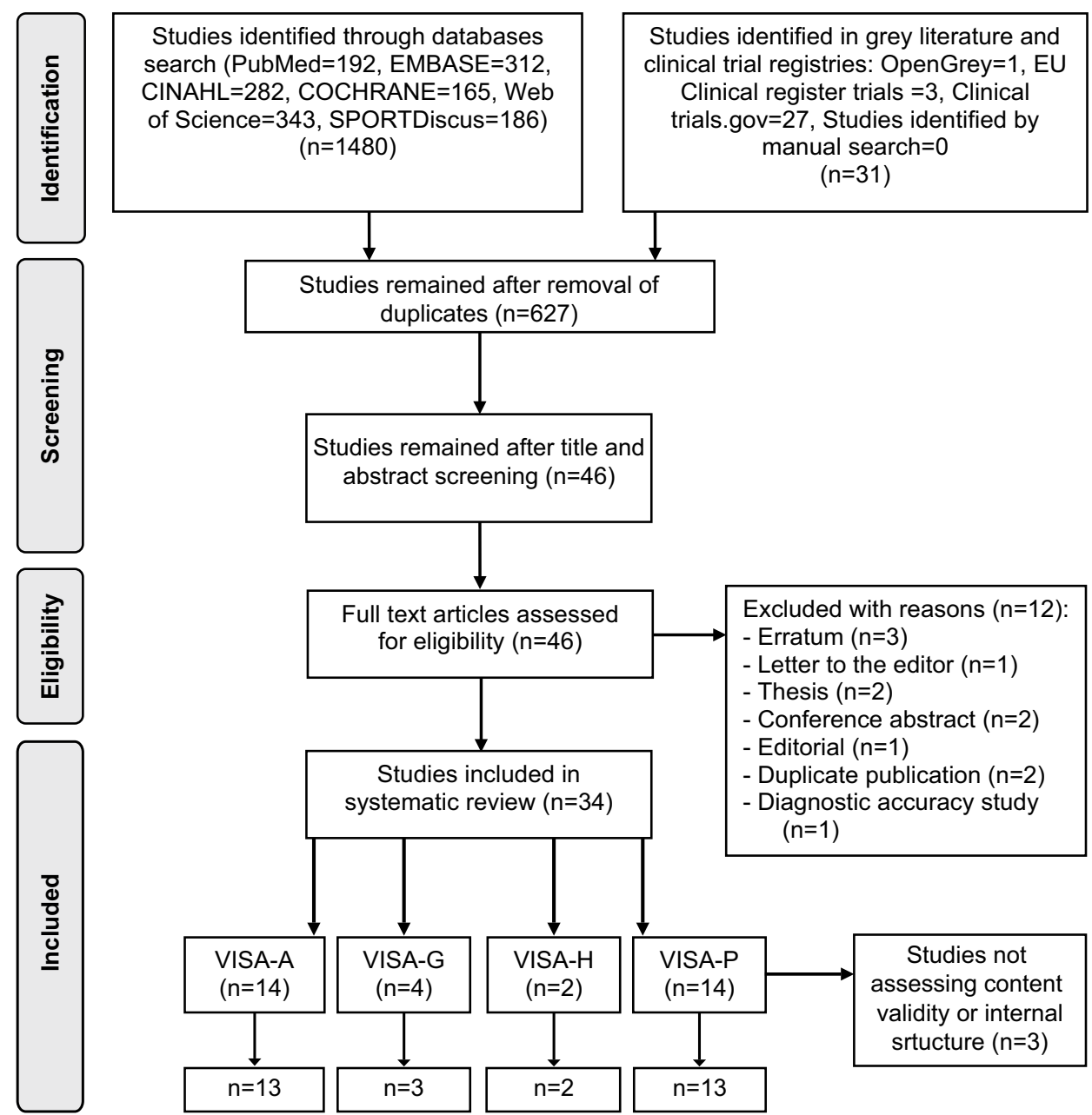




\section{VISA-H}

Very low quality of evidence was found for VISA-H (Table 2).

\section{VISA-P}

Four cross-cultural adaptations $[25,27,46,81]$ did not evaluate content validity. Very-low-quality evidence was found for inconsistent content validity of VISA-P (Table 2).

\section{Quality, results, and evidence synthesis of studies evaluating structural validity and internal consistency}

\section{VISA-A}

Low-quality evidence indicated sufficient unidimensionality for VISA-A (Tables 2, 3).

Sufficient internal consistency of the unidimensional VISA-A was found (Table 3). The pooled Cronbach's $\alpha$ using a random-effects model was 0.79 (Fig. 2). By subgrouping the studies that included only patients with Achilles tendinopathy or a mixed group of patients and asymptomatic individuals, the pooled estimate for alpha was 0.74 (95\% CI $0.68-0.80$ ) for patients and 0.87 (95\% CI 0.82-0.92) for the mixed group. As the quality of evidence for internal consistency cannot be higher than the quality of evidence for structural validity [64], low-quality evidence suggests sufficient internal consistency for VISA-A.
VISA-G

Indeterminate evidence was found for VISA-G structural validity and internal consistency (criteria for "at least low evidence for sufficient structural validity" were not met [64]) (Tables 2, 3).

\section{VISA-H}

Low-quality evidence was found for insufficient unidimensionality of VISA-H and the results for internal consistency were ignored due to evidence of a 2-factor structure (Tables 2, 3).

\section{VISA-P}

Conflicting results were found on the structure of the VISA$\mathrm{P}$, this inconsistency could not be explained, and the evidence was not graded (Table 2).

Internal consistency received an indeterminate rating (?) due to inconsistent results for structural validity (Tables 2, 3) [64].

\section{Quality and results of studies evaluating cross-cultural validity/measurement invariance}

No studies evaluated cross-cultural validity/measurement invariance of the VISA-A, VISA-G, or VISA-H. Only one study [25] of doubtful quality examined measurement invariance of VISA-P across sexes using multi-group confirmatory factor analysis. The difference of comparative fit index values was $<0.01$ [9], indicating that VISA-P scores are comparable between men and women. However, examination
Table 1 COSMIN quality evaluation of the VISA content validity studies

\begin{tabular}{|c|c|c|c|c|c|c|}
\hline \multirow[t]{2}{*}{ Study } & \multirow[t]{2}{*}{ PROM } & \multicolumn{3}{|c|}{ Asking patients } & \multicolumn{2}{|c|}{ Asking experts } \\
\hline & & Relevance & $\begin{array}{l}\text { Compre- } \\
\text { hensive- } \\
\text { ness }\end{array}$ & $\begin{array}{l}\text { Compre- } \\
\text { hensibility }\end{array}$ & Relevance & $\begin{array}{l}\text { Compre- } \\
\text { hensive- } \\
\text { ness }\end{array}$ \\
\hline Silbernagel et al. [71] & VISA-A & & & $\mathrm{D}$ & & \\
\hline Dogramaci et al. [18] & VISA-A & $\mathrm{D}$ & & $\mathrm{D}$ & & \\
\hline Kaux et al. [33] & VISA-A & & & $\mathrm{D}$ & & \\
\hline De Mesquita et al. [16] & VISA-A & & & & $\mathrm{D}$ & \\
\hline Hernandez-Sanchez et al. [28] & VISA-A & & & $\mathrm{D}$ & & \\
\hline Beaudart et al. [3] & VISA-G & & & $\mathrm{D}$ & & \\
\hline Jorgensen et al. [31] & VISA-H & & & $\mathrm{D}$ & & \\
\hline Locquet et al. [40] & VISA-H & & & $\mathrm{D}$ & & \\
\hline Hernandez-Sanchez et al. [26] & VISA-P & & & $\mathrm{D}$ & & \\
\hline Lohrer et al. [42] & VISA-P & & & & I & \\
\hline Korakakis et al. [36] & VISA-P & & & & $\mathrm{D}$ & \\
\hline Acharya et al. [1] & VISA-P & & & $\mathrm{D}$ & & \\
\hline
\end{tabular}

$A$ Achilles, $D$ doubtful, $G$ greater trochanteric pain syndrome, $H$ hamstring, $I$ inadequate 


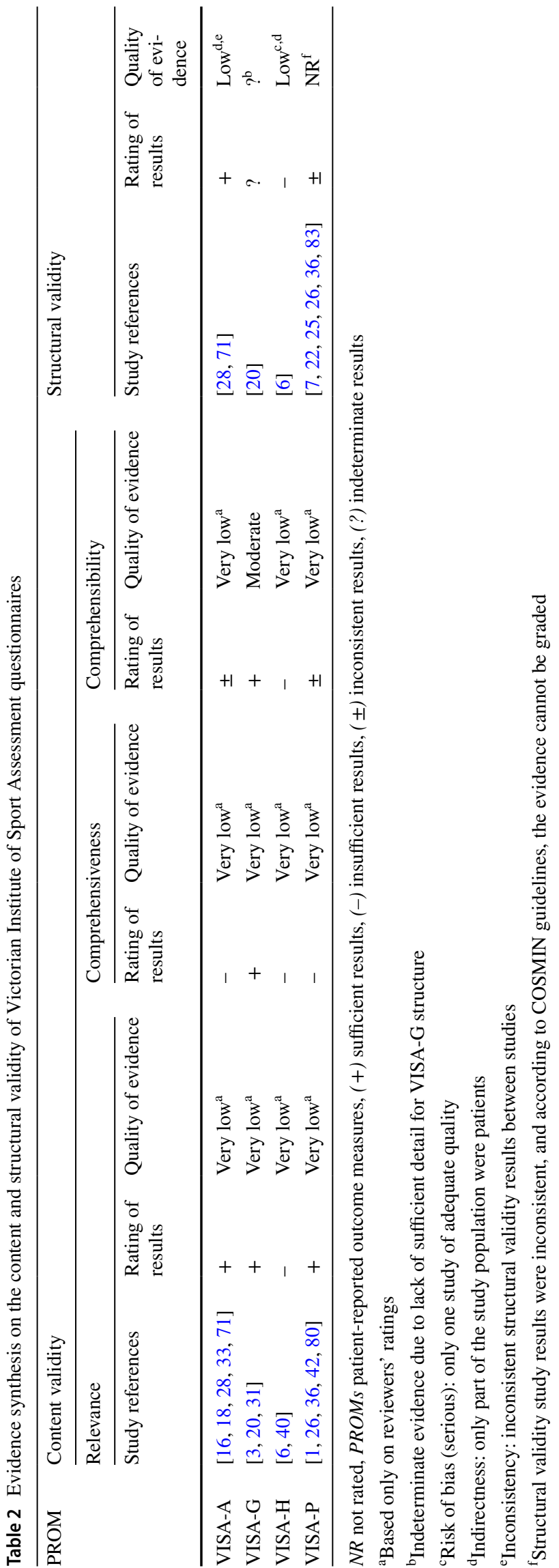

of measurement invariance was performed in a model that did not met the requirements of sufficient unidimensionality $[10,64]$. Low-quality evidence (very serious risk of bias) indicates sufficient measurement invariance between sexes for VISA-P.

\section{Internal structure in patients with other conditions}

One study [43] of doubtful quality evaluated internal consistency of VISA-A in 39 patients with Haglund's deformity providing indeterminate evidence as there is no information on the structural validity of the questionnaire in this population.

\section{Discussion}

The most important finding of the present study was the very-low-quality evidence for the VISA questionnaires' content and structural validity in assessing the severity of symptoms and disability in patients with lower limb tendinopathies.

More specifically, in relation to content validity, VISA-A presented very-low-quality sufficient relevance, insufficient comprehensiveness, and inconsistent comprehensibility. The VISA-G displayed moderate-quality evidence for sufficient comprehensibility and very-low-quality evidence of sufficient relevance and comprehensiveness. The VISA-P presented very-low-quality sufficient relevance, insufficient comprehensiveness, and inconsistent comprehensibility, while VISA-H presented very-low-quality evidence of insufficient content validity.

VISA-A displayed low-quality evidence for sufficient unidimensionality and internal structure, while for VISA-G, the rating was indeterminate. VISA-H presented low-quality evidence of insufficient unidimensionality. The structural validity of the VISA-P was inconsistent. Internal consistency for VISA-G, VISA-H, and VISA-P was indeterminate. Lowquality evidence from limited available data indicates sufficient measurement invariance between sexes for VISA-P.

\section{Content validity}

The VISA questionnaires are routinely used as a core outcome measure in tendinopathy research and clinical practice $[44,52,77,78]$. Content validity is the degree to which the content of an instrument is an adequate reflection of the construct to be measured [55]. The inadequate quality evidence supporting the content validity of the VISAs is unfortunately similar to other musculoskeletal questionnaires [10]. A lack of content validity affects all other measurement properties [74]. A recent consensus [65] recommended that at least content validity and internal structure should be adequate 


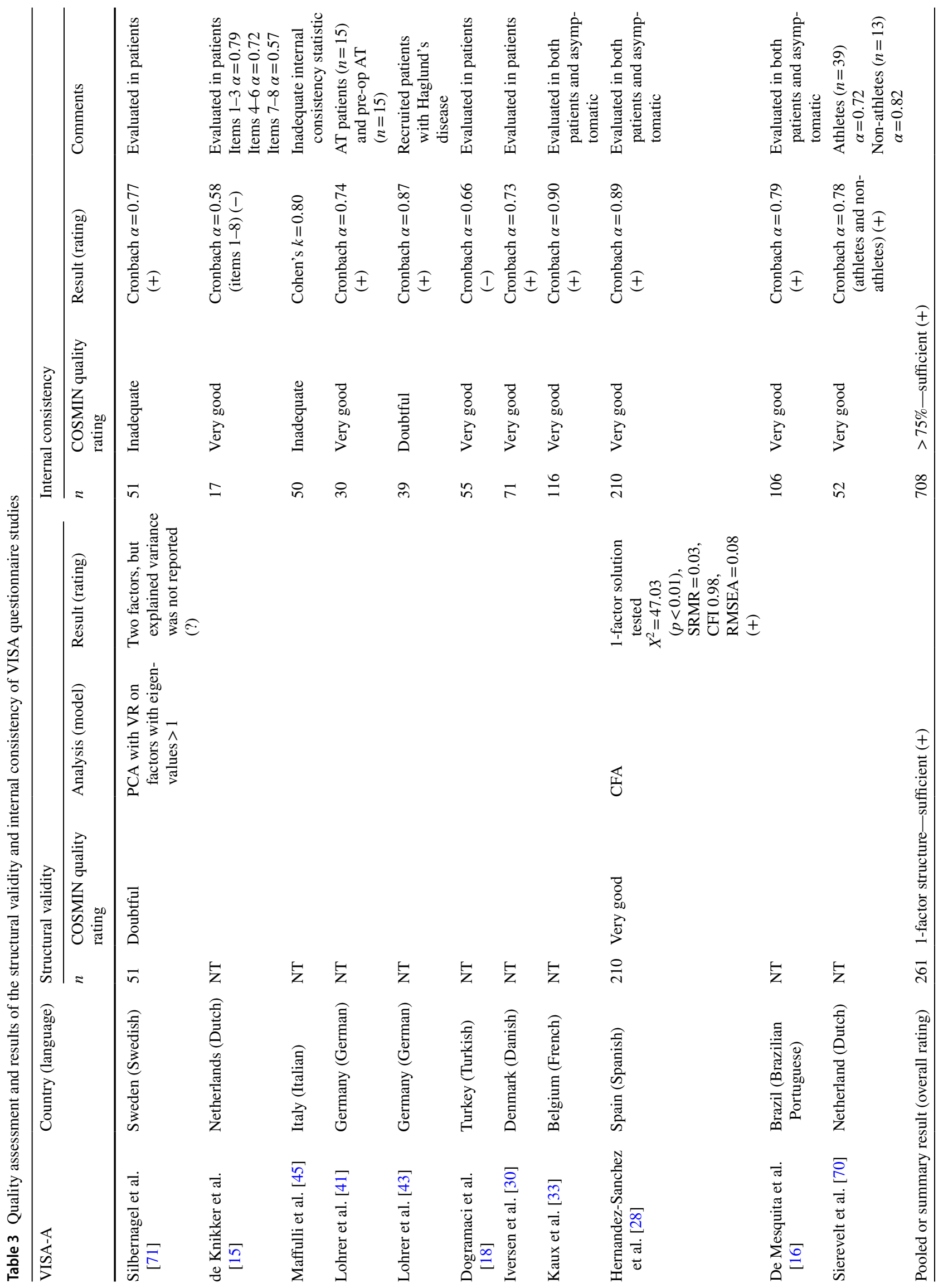




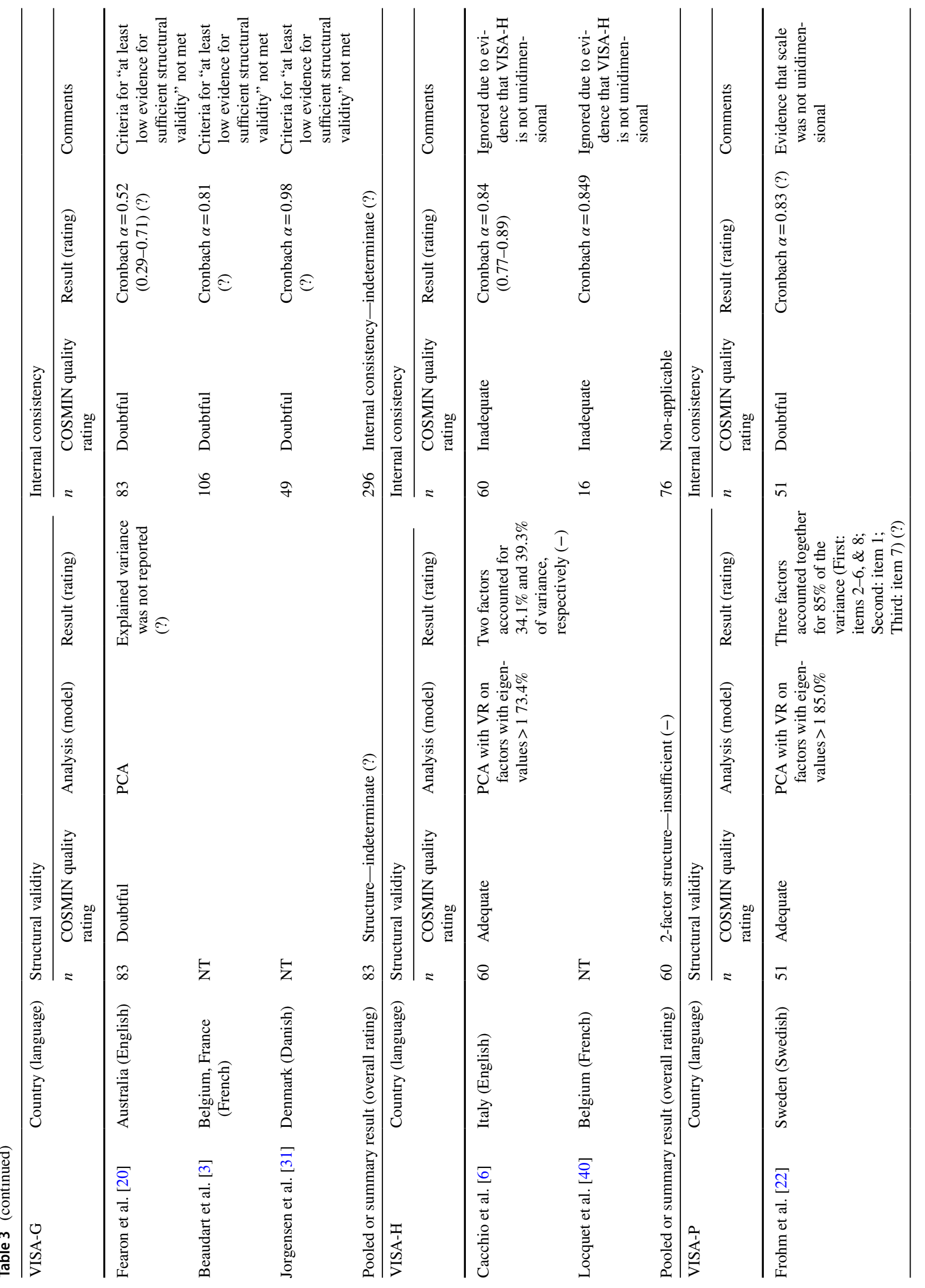




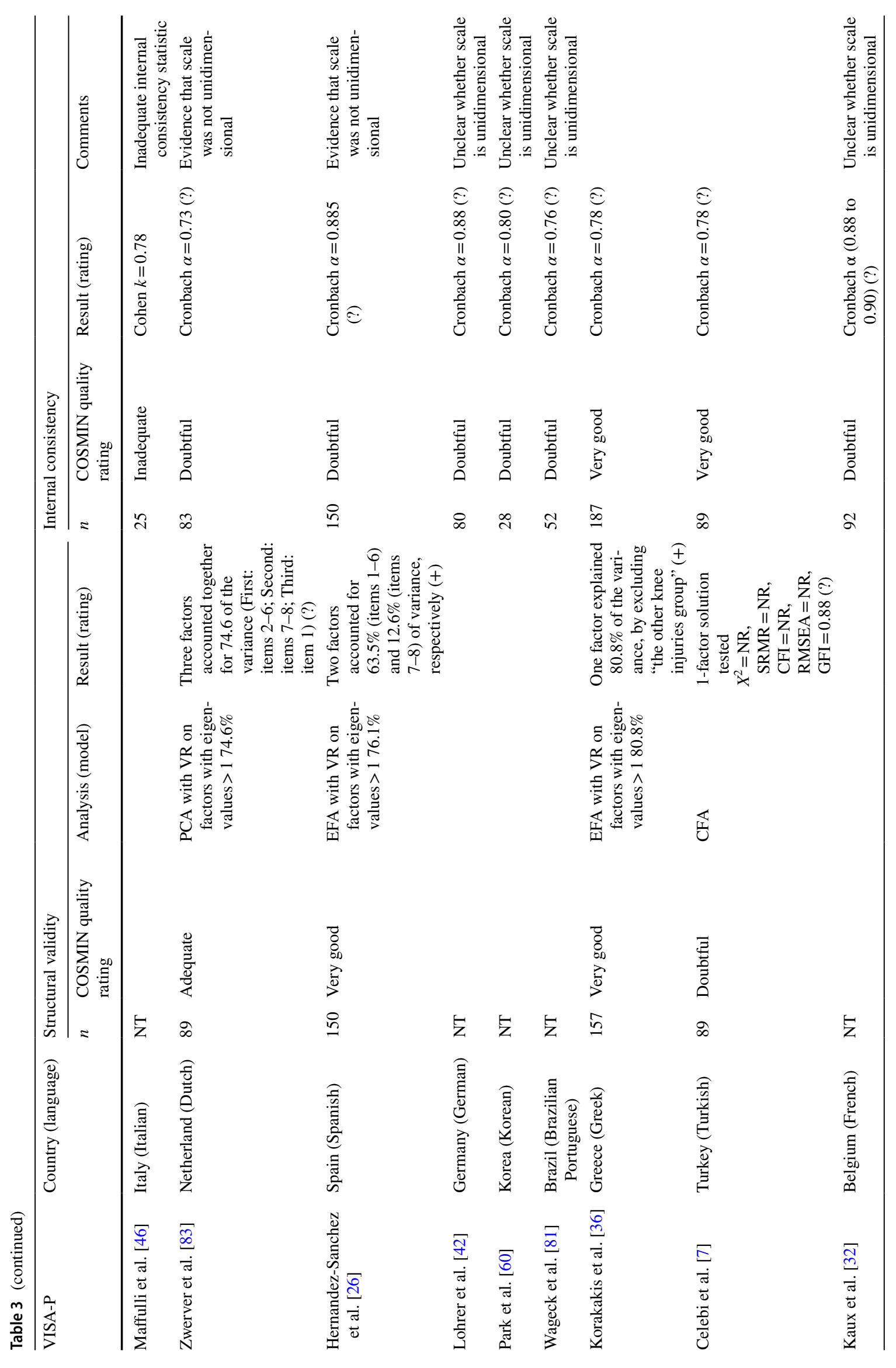




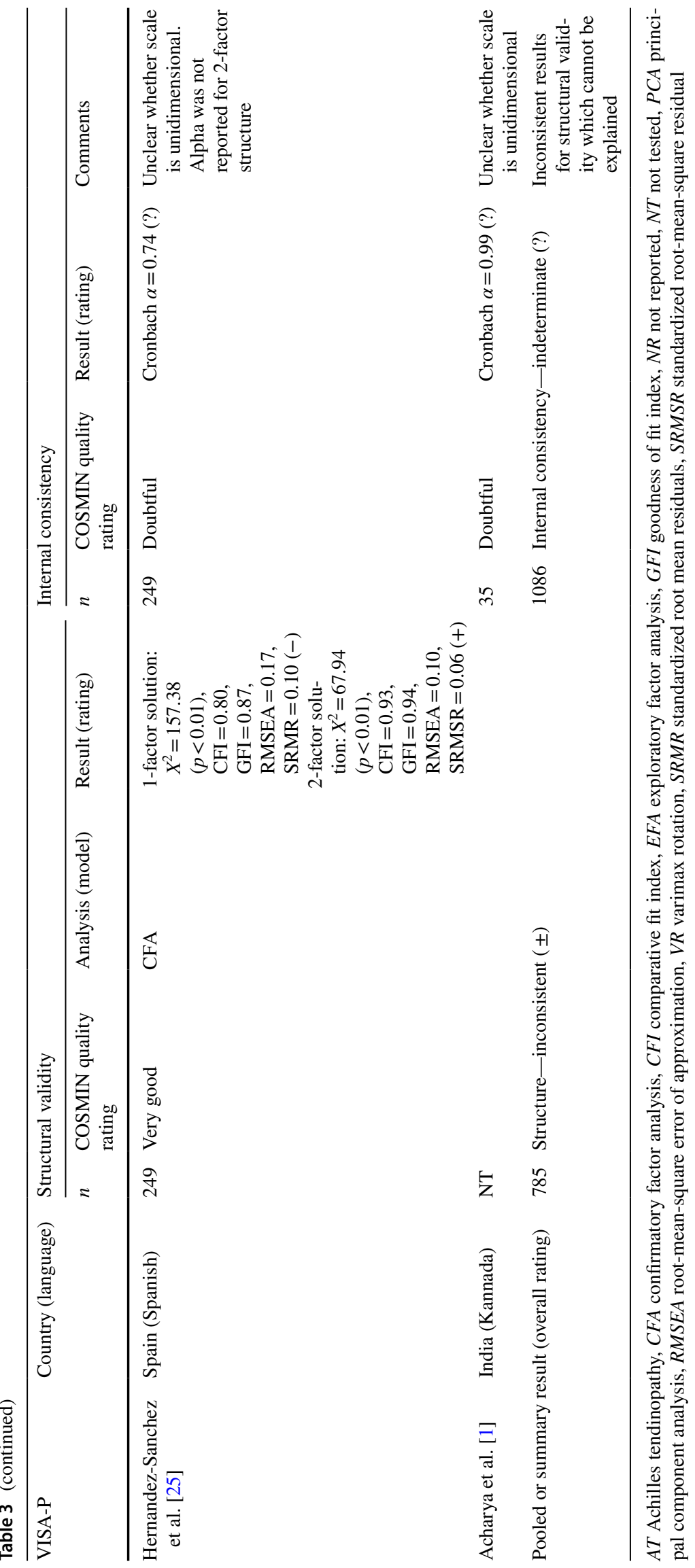


Fig. 2 Forest plot of pooled Cronbach's $\alpha$ coefficient for the Victorian Institute of Sport Assessment scale-Achilles (VISA-A). $C I$ confidence intervals

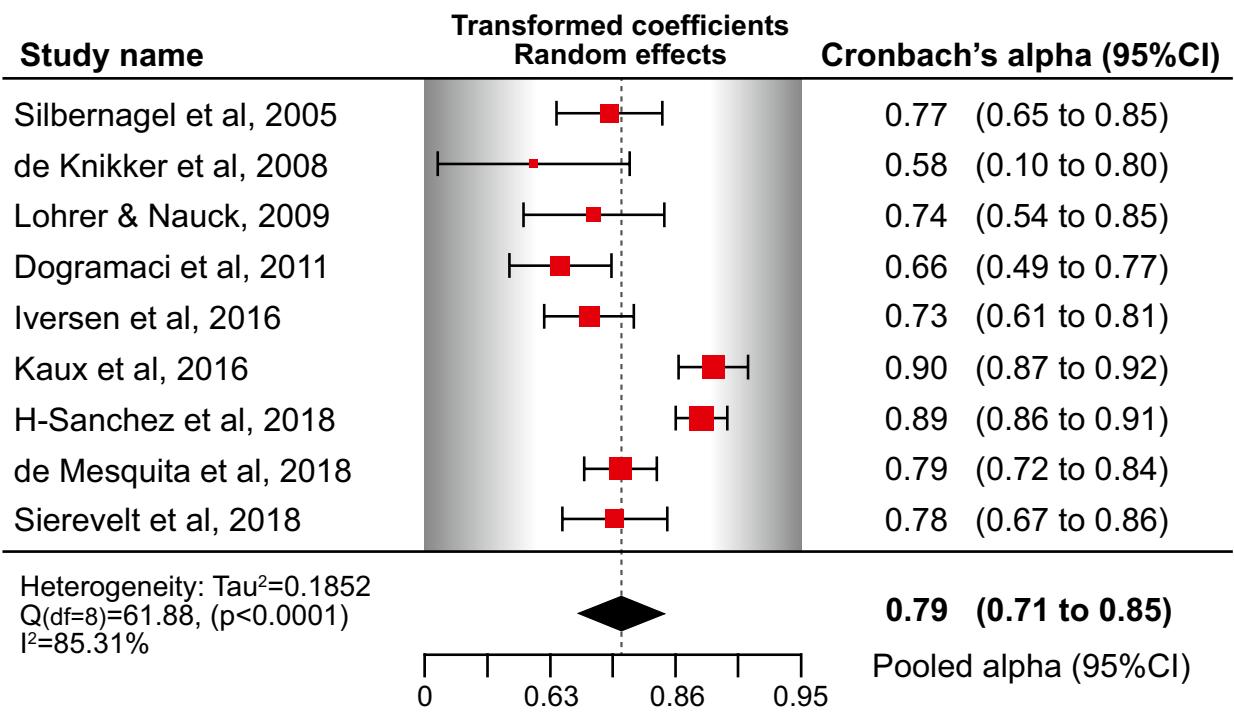

patient-oriented qualitative exploration. Only the development of VISA-G included an adequate number of patients, but was limited to the assessment of comprehensibility. Mounting evidence suggests an association of psychological variables and outcome in tendinopathy, highlighting the need to address, from the patient's perspective, the psychosocial factors in the evaluation of tendinopathy $[48,50$, $63,75]$. Clinical research and empirical evidence have also underpinned other key features of tendinopathy that could plausibly be relevant for the construct of interest or replace existing items of the VISAs. For example, energy-storage and release activities, increases of the magnitude or rate of application of loading decline squat for patellar tendinopathy, or countermovement jump for Achilles tendinopathy are usually seen to increase symptoms in tendinopathy patients [44, 47, 68]. Important aspects of tendinopathy may be missing in the VISA questionnaires. It is suggested that the relevance as well as the comprehensiveness of the VISAs items require update and further investigation considering the current understanding of tendinopathy [49].

\section{Dimensionality and construct validity}

Construct validity is the degree to which the scores of the PROM are consistent with predetermined hypotheses based on the assumption that the PROM validly measures the construct of interest [55]. In turn, structural validity is the degree to which the scores of a PROM are an adequate reflection of the dimensionality of the construct [55]. The VISA-A, VISA-H, and VISA-P questionnaires were formulated based on the hypotheses that the PROM will measure symptoms, function, and ability of patients to undertake sports as the domains of the same construct $[6,69,80]$, while VISA-G was formulated to assess the severity of disability associated with greater trochanteric 
pain syndrome [20]. Interestingly, concerns were raised by this review as the VISA questionnaires do not share the same quality evidence for their underlying structure. Additionally, evidence suggests that the VISA questionnaires measure more than one construct and present violations of the assumptions of unidimensionality (Table 3). As such, a firm conclusion could not be reached with high-quality evidence regarding the underlying structure of the questionnaires. The 2-factor structure reported in development or cross-cultural adaptation studies (VISA-A, VISA-H, and VISA-P) included one or two items, mostly related with the physical activity section, suggesting that for the measurement of a broader, or a second construct important items maybe missing. From a different perspective, the scoring of the physical activity or sports participation section has been argued to substantially affect the scoring and consequently the underlying structure of the PROMs [49]. The VISA-A, VISA-H, and VISA-P were primarily designed for sporting populations; however, tendinopathy also occurs in non-sporting or sedentary individuals [14]. Item 8 ("sports") of the VISAs is irrelevant to nonsporting/inactive individuals (e.g., greater trochanteric pain syndrome patients, sedentary individuals) $[20,67$, 70]. This results in sedentary individuals scoring 0 in both items 7 and 8 and an underestimation of the total score irrespective of their symptoms. Inversely, the heavily weighted items 7 and $8(40 / 100)$ in the overall scoring lead to an overestimation of the total score in high-level athletes that continue training with symptoms [49]. Moreover, the scoring formula of item $7(0,4,7$, or 10 points $)$ has been argued to affect the variability of the scores, thus affecting the dimensionality analysis [25]. Modified versions of the VISA have been proposed or modifications of scoring of item 7 have been suggested to overcome this issue $[25,56,67,70]$.

According to COSMIN guidelines [54], evidence for structural validity is a prerequisite for the internal consistency and cross-cultural validity/measurement invariance of a PROM. Cronbach's $\alpha$ can only be interpreted as a measure of internal consistency when the scale or subscale is unidimensional [12, 54]. Only the VISA-A displayed sufficient unidimensionality with acceptable internal consistency among its items; however, with low-quality evidence. These results were consistent with a recent reliability generalization meta-analysis of five studies reporting an alpha ranging from 0.70 to 0.79 [58]. Conversely, the internal consistency of the VISA-G, VISA-H, and VISA-P could not be rated due to inconsistent and indeterminate structural validity, and the lack of reporting of an alpha coefficient for each of the 2-factor structures, respectively. Given that when the assumption of unidimensionality is not met or evaluated the Cronbach's $\alpha$ may overestimate the true internal consistency, pooled coefficients for VISA-P should be interpreted with extreme caution [59], and clinicians and researchers should be encouraged not to use the total scores of the PROM [54, 82].

Structural validity or measurement invariance requires that the items quantifying a construct of interest function in the same way across groups (e.g., between different cultures or genders) [23]. For example, significant gender differences in response to 12 weeks' eccentric training in patients with Achilles tendinopathy have been documented [35]. It is currently not possible to determine if these are artefacts of the questionnaire or biological in nature [4]. It was confirmed by our review that measurement invariance is tested relatively infrequently in musculoskeletal research [23]. Low-quality evidence of measurement invariance was found, indicating that only VISA-P scores are comparable between Spanish men and women [25]. Future studies evaluating invariance of factor structure of VISA questionnaires across groups are much needed. Sparse information was available on the methodology and results of most included content validity studies. Future PROM development studies should: explain the item obtention and reduction method, prove pilot testing was conducted, and consult the COSMIN guidelines [74] on this measurement property. Finally, patient involvement in content validity studies is essential-the relevance and the comprehensiveness of the VISA questionnaires are yet to be adequately explored.

The validation process of the VISAs included only classical test theory methods, although approaches such as Rasch analysis have been advocated as more robust and useful in the evaluation of unidimensional PROMs [38]. Rasch analysis effectively evaluates the relevance and contribution of each item in measuring the underlying construct, the appropriateness of the response categories, and the amount of construct targeted by each item-overlooked properties in VISA validation studies. Rasch analysis should explore the unidimensionality of the VISA questionnaires. If it is violated, a refinement of the PROMs should be implemented by including the entire spectrum of the disease in their items [74].

A degree of subjectivity was necessary in the rating of the standards of the criteria of this newly formed guidelines, though the involvement of three reviewers and the pre-specified criteria helped to minimize the possibility of bias. Also, a weakness of our review was the consideration of different language versions of the PROMs as the same questionnaire in the evidence syntheses. However, this methodology has been recommended [74] and previously used [10]. Finally, COSMIN is an early set of guidelines with acknowledged limitations [64] that have to be evaluated in future research.

As suggested by patients and healthcare professionals from the International Scientific Tendinopathy Symposium Consensus, disability is among the nine domains of the core outcome set for tendinopathy. The VISA questionnaires have 
been recommended in research and clinical practice, because they are condition-specific composite scores of a mix of patient-rated pain and disability due to the pain, usually relating to tendon-specific activities [78]. Based on the COSMIN standards, none of the VISA questionnaires met the requirements to be rated as a category "A" PROM (recommended for use and the results obtained can be trusted) [64]. All VISA questionnaires were categorized as "B" PROMs, meaning that may have the potential to be recommended, but further validation studies are needed to assess their quality.

\section{Conclusion}

Given the lack of alternative condition-specific outcome measures, we recommend the use of the VISAs in their current form, but the results obtained from their use should be interpreted with caution, especially for VISA-A, VISA$\mathrm{H}$, and VISA-P that presented insufficient or inconsistent ratings in content and structural validity. Researchers and clinicians should be using the VISA questionnaires in conjunction with other joint specific PROMs to capture the multifaceted presentation of the lower limb tendinopathies more adequately.

Supplementary Information The online version contains supplementary material available at https://doi.org/10.1007/s00167-021-06598-5.

Author contribution All authors contributed to this work and the authorship of this manuscript. VK planned, coordinated the idea, conducted the search, analyzed results, and wrote and reviewed the manuscript; AK, MS, and RW coordinated the idea, analyzed the results, provided writing content, and reviewed; YS conducted the search, and wrote and reviewed the manuscript; and KT provided writing, editing, and review support.

Funding Open access funding provided by the Qatar National Library.

\section{Declarations}

Conflict of interest The authors have no relevant financial or non-financial interests to disclose.

Funding No funding was received for conducting this review.

Ethical approval Not applicable.

Informed consent Not applicable.

Open Access This article is licensed under a Creative Commons Attribution 4.0 International License, which permits use, sharing, adaptation, distribution and reproduction in any medium or format, as long as you give appropriate credit to the original author(s) and the source, provide a link to the Creative Commons licence, and indicate if changes were made. The images or other third party material in this article are included in the article's Creative Commons licence, unless indicated otherwise in a credit line to the material. If material is not included in the article's Creative Commons licence and your intended use is not permitted by statutory regulation or exceeds the permitted use, you will need to obtain permission directly from the copyright holder. To view a copy of this licence, visit http://creativecommons.org/licenses/by/4.0/.

\section{References}

1. Acharya GU, Kumar A, Rajasekar S, Samuel AJ (2019) Reliability and validity of Kannada version of Victorian Institute of Sports Assessment for patellar tendinopathy (VISA-P-K) questionnaire. J Clin Orthop Trauma 10:S189-S192

2. Beaton DE, Bombardier C, Guillemin F, Ferraz MB (2000) Guidelines for the process of cross-cultural adaptation of self-report measures. Spine 25:3186-3191

3. Beaudart $\mathrm{C}$, Gillier $\mathrm{M}$, Bornheim $\mathrm{S}$, Van Beveren $\mathrm{J}$, Bruyère $\mathrm{O}$, Kaux JF (2020) French translation and validation of the "Victorian Institute of Sports Assessment for Gluteal Tendinopathy" questionnaire. PM\&R. https://doi.org/10.1002/pmrj.12391

4. Beckstead JW, Yang C-Y, Lengacher CA (2008) Assessing crosscultural validity of scales: a methodological review and illustrative example. Int J Nurs Stud 45:110-119

5. Brod M, Tesler LE, Christensen TL (2009) Qualitative research and content validity: developing best practices based on science and experience. Qual Life Res 18:1263-1278

6. Cacchio A, De Paulis F, Maffulli N (2014) Development and validation of a new visa questionnaire (VISA-H) for patients with proximal hamstring tendinopathy. Br J Sports Med 48:i39-i44

7. Celebi MM, Kose SK, Akkaya Z, Zergeroglu AM (2016) Crosscultural adaptation of VISA-P score for patellar tendinopathy in Turkish population. Springerplus 5:1453

8. Chen PC, Wu KT, Chou WY, Huang YC, Wang LY, Yang TH et al (2019) Comparative effectiveness of different nonsurgical treatments for patellar tendinopathy: a systematic review and network meta-analysis. Arthroscopy 35:3117-3131.e3112

9. Cheung GW, Rensvold RB (2002) Evaluating goodness-of-fit indexes for testing measurement invariance. Struct Equ Model 9:233-255

10. Chiarotto A, Ostelo RW, Boers M, Terwee CB (2018) A systematic review highlights the need to investigate the content validity of patient-reported outcome measures for physical functioning in patients with low back pain. J Clin Epidemiol 95:73-93

11. Clifford C, Challoumas D, Paul L, Syme G, Millar NL (2020) Effectiveness of isometric exercise in the management of tendinopathy: a systematic review and meta-analysis of randomised trials. BMJ Open Sport Exerc Med 6:e000760

12. Cortina JM (1993) What is coefficient alpha? An examination of theory and applications. J Appl Psychol 78:98-104

13. COSMIN. COnsensus-based Standards for the selection of health Measurement INstruments (COSMIN) http://www.cosmin.nl. Accessed 18 May 2020

14. de Jonge S, van den Berg C, de Vos RJ, van der Heide HJ, Weir A, Verhaar JA et al (2011) Incidence of midportion Achilles tendinopathy in the general population. Br J Sports Med 45:1026-1028

15. de Knikker R, El Hadouchi M, Velthuis M, Wittink H (2008) The Dutch version of the Victoria Institute of Sports AssessmentAchilles (VISA-A) questionnaire: cross-cultural adaptation and validation in Dutch patients with Achilles tendinopathy. Nederlands Tijdschrift Voor Fysiotherapie 118:34-41

16. de Mesquita GN, de Oliveira MNM, Matoso AER, de Moura AG, de Oliveira RR (2018) Cross-cultural adaptation and Measurement Properties of the Brazilian Portuguese Version of the Victorian Institute of Sport Assessment-Achilles (VISA-A) Questionnaire. J Orthop Sports Phys Ther 48:567-573 
17. de Vet HC, Adèr HJ, Terwee CB, Pouwer F (2005) Are factor analytical techniques used appropriately in the validation of health status questionnaires? A systematic review on the quality of factor analysis of the SF-36. Qual Life Res 14:1203-1218 (discussion 1219-1221)

18. Dogramaci Y, Kalaci A, Kucukkubas N, Inandi T, Esen E, Yanat AN (2011) Validation of the VISA-A questionnaire for Turkish language: the VISA-A-Tr study. Br J Sports Med 45:453-455

19. Ebert JR, Fearon AM, Smith A, Janes GC (2019) Responsiveness of the Victorian Institute for Sport Assessment for Gluteal Tendinopathy (VISA-G), modified Harris hip and Oxford hip scores in patients undergoing hip abductor tendon repair. Musculoskelet Sci Pract 43:1-5

20. Fearon AM, Ganderton C, Scarvell JM, Smith PN, Neeman T, Nash C et al (2015) Development and validation of a VISA tendinopathy questionnaire for greater trochanteric pain syndrome, the VISA-G. Man Ther 20:805-813

21. Floyd FJ, Widaman KF (1995) Factor analysis in the development and refinement of clinical assessment instruments. Psychol Assess 7:286-299

22. Frohm A, Saartok T, Edman G, Renström P (2004) Psychometric properties of a Swedish translation of the VISA-P outcome score for patellar tendinopathy. BMC Musculoskelet Disord $5: 49$

23. Gregorich SE (2006) Do self-report instruments allow meaningful comparisons across diverse population groups? Testing measurement invariance using the confirmatory factor analysis framework. Med Care 44:S78-94

24. Hakstian AR, Whalen TE (1976) A k-sample significance test for independent alpha coefficients. Psychometrika 41:219-231

25. Hernandez-Sanchez S, Abat F, Hidalgo MD, Cuesta-Vargas AI, Segarra V, Sanchez-Ibañez JM et al (2017) Confirmatory factor analysis of VISA-P scale and measurement invariance across sexes in athletes with patellar tendinopathy. J Sport Health Sci 6:365-371

26. Hernandez-Sanchez S, Hidalgo MD, Gomez A (2011) Crosscultural adaptation of VISA-P score for patellar tendinopathy in Spanish population. J Orthop Sports Phys 41:581-591

27. Hernandez-Sanchez S, Hidalgo MD, Gomez A (2014) Responsiveness of the VISA-P scale for patellar tendinopathy in athletes. Br J Sports Med 48:i32-i38

28. Hernandez-Sanchez S, Poveda-Pagan EJ, Alakhdar-Mohmara Y, Hidalgo MD, Fernandez-De-Las-Penas C, Arias-Buria JL (2018) Cross-cultural adaptation of the Victorian Institute of Sport Assessment-Achilles (VISA-A) Questionnaire for Spanish Athletes with Achilles tendinopathy. J Orthop Sports Phys 48:111-120

29. Higgins JPT, Green S (2011) Cochrane Handbook for Systematic Reviews of Interventions. Version 5.1.0 ed. The Cochrane Collaboration

30. Iversen JV, Bartels EM, Jørgensen JE, Nielsen TG, Ginnerup C, Lind MC et al (2016) Danish VISA - a questionnaire with validation and reliability testing for Danish-speaking Achilles tendinopathy patients. Scand J Med Sci Sports 26:1423-1427

31. Jorgensen JE, Fearon AM, Molgaard CM, Kristinsson J, Andreasen J (2020) Translation, validation and test-retest reliability of the VISA-G patient-reported outcome tool into Danish (VISA-G.DK). PeerJ 8:14

32. Kaux JF, Delvaux F, Oppong-Kyei J, Beaudart C, Buckinx F, Croisier JL et al (2016) Cross-cultural adaptation and validation of the Victorian Institute of Sport Assessment-Patella Questionnaire for French-speaking patients with patellar tendinopathy. J Orthop Sports Phys 46:384-390

33. Kaux JF, Delvaux F, Oppong-Kyei J, Dardenne N, Beaudart C, Buckinx F et al (2016) Validity and reliability of the French translation of the VISA-A Questionnaire for Achilles tendinopathy. Disabil Rehabil 38:2593-2599

34. Keller A, Wagner P, Izquierdo G, Cabrolier J, Caicedo N, Wagner E et al (2018) Cross-cultural adaptation and validation of the VISA-A Questionnaire for Chilean Spanish-speaking patients. J Orthop Surg Res 13:177

35. Knobloch K, Schreibmueller L, Kraemer R, Jagodzinski M, Vogt PM, Redeker J (2010) Gender and eccentric training in Achilles mid-portion tendinopathy. Knee Surg Sports Traumatol Arthrosc 18:648-655

36. Korakakis V, Patsiaouras A, Malliaropoulos N (2014) Crosscultural adaptation of the VISA-P questionnaire for Greekspeaking patients with patellar tendinopathy. Br J Sports Med 48:1647-1652

37. Korakakis V, Whiteley R, Tzavara A, Malliaropoulos N (2018) The effectiveness of extracorporeal shockwave therapy in common lower limb conditions: a systematic review including quantification of patient-rated pain reduction. Br J Sports Med 52:387-407

38. Leung YY, Png ME, Conaghan P, Tennant A (2014) A systematic literature review on the application of Rasch analysis in musculoskeletal disease-a special interest group report of OMERACT 11. J Rheumatol 41:159-164

39. Liberati A, Altman DG, Tetzlaff J, Mulrow C, Gøtzsche PC, Ioannidis JPA et al (2009) The PRISMA statement for reporting systematic reviews and meta-analyses of studies that evaluate healthcare interventions: explanation and elaboration. BMJ 339:b2700

40. Locquet $\mathrm{M}$, Bornheim S, Colas L, Van Beveren J, Bruyère O, Reginster JY et al (2019) French translation of the Victorian Institute of Sport Assessment Scale for proximal hamstring tendinopathy (VISA-H). J Traumatol Sport 36:217-221

41. Lohrer H, Nauck T (2009) Cross-cultural adaptation and validation of the VISA-A questionnaire for German-speaking Achilles tendinopathy patients. BMC Musculoskelet Disord 10:134

42. Lohrer H, Nauck T (2011) Cross-cultural adaptation and validation of the VISA-P Questionnaire for German-speaking patients with patellar tendinopathy. J Orthop Sports Phys 41:180-190

43. Lohrer H, Nauck T (2010) Validation of the VISA-A-G Questionnaire for German-speaking patients suffering from Haglund's disease. Sportverletz Sportschaden 24:98-106

44. Macdermid JC, Silbernagel KG (2015) Outcome evaluation in tendinopathy: foundations of assessment and a summary of selected measures. J Orthop Sports Phys 45:950-964

45. Maffulli N, Longo UG, Testa V, Oliva F, Capasso G, Denaro V (2008) Italian translation of the VISA-A score for tendinopathy of the main body of the Achilles tendon. Disabil Rehabil 30:1635-1639

46. Maffulli N, Longo UG, Testa V, Oliva F, Capasso G, Denaro V (2008) VISA-P score for patellar tendinopathy in males: adaptation to Italian. Disabil Rehabil 30:1621-1624

47. Malliaras P, Cook J, Purdam C, Rio E (2015) Patellar tendinopathy: clinical diagnosis, load management, and advice for challenging case presentations. J Orthop Sports Phys Ther 45:887-898

48. Mallows A, Debenham J, Walker T, Littlewood C (2017) Association of psychological variables and outcome in tendinopathy: a systematic review. Br J Sports Med 51:743-748

49. Mallows A, Littlewood C, Malliaras P (2018) Measuring patientreported outcomes (PROs/PROMs) in people with Achilles tendinopathy: how useful is the VISA-A? Br J Sports Med 52:1221

50. Mc Auliffe S, Synott A, Casey H, Mc Creesh K, Purtill H, O'Sullivan K (2017) Beyond the tendon: experiences and perceptions of people with persistent Achilles tendinopathy. Musculoskelet Sci Pract 29:108-114

51. McCormack J, Underwood F, Slaven E, Cappaert T (2015) The minimum clinically important difference on the VISA-A and LEFS for patients with insertional Achilles tendinopathy. Int $\mathbf{J}$ Sports Phys Ther 10:639-644 
52. Mendonca LM, Leite HR, Zwerver J, Henschke N, Branco G, Oliveira VC (2020) How strong is the evidence that conservative treatment reduces pain and improves function in individuals with patellar tendinopathy? A systematic review of randomised controlled trials including GRADE recommendations. Br J Sports Med 54:87-93

53. Moher D, Liberati A, Tetzlaff J, Altman DG (2009) Preferred reporting items for systematic reviews and meta-analyses: the PRISMA statement. BMJ 339:332-336

54. Mokkink LB, de Vet HCW, Prinsen CAC, Patrick DL, Alonso J, Bouter LM et al (2018) COSMIN risk of bias checklist for systematic reviews of patient-reported outcome measures. Qual Life Res 27:1171-1179

55. Mokkink LB, Terwee CB, Patrick DL, Alonso J, Stratford PW, Knol DL et al (2010) The COSMIN study reached international consensus on taxonomy, terminology, and definitions of measurement properties for health-related patient-reported outcomes. J Clin Epidemiol 63:737-745

56. Munteanu SE, Scott LA, Bonanno DR, Landorf KB, Pizzari T, Cook JL et al (2015) Effectiveness of customised foot orthoses for Achilles tendinopathy: a randomised controlled trial. Br J Sports Med 49:989-994

57. Nauwelaers AK, Van Oost L, Peers K (2020) Evidence for the use of PRP in chronic midsubstance Achilles tendinopathy: a systematic review with meta-analysis. Foot Ankle Surg. https://doi.org/ 10.1016/j.fas.2020.07.009

58. Palazón-Bru A, Mares-García E, Gil-Guillén VF, Tomás-Rodríguez MI (2019) A reliability generalization meta-analysis of the Victorian Institute of Sport Assessment Scale for Achilles tendinopathy (VISA-A). Foot Ankle Int 40:430-438

59. Palazón-Bru A, Tomás Rodríguez MI, Mares-García E, Hernández-Sánchez S, Carbonell-Torregrosa M, Gil-Guillén VF (2020) The Victorian Institute of Sport Assessment Scale for Patellar tendinopathy (VISA-P): a reliability generalization meta-analysis. Clin J Sport Med. https://doi.org/10.1097/jsm.0000000000000810

60. Park BH, Seo JH, Ko MH, Park SH (2013) Reliability and validity of the Korean version VISA-P questionnaire for patellar tendinopathy in adolescent elite volleyball athletes. Ann Rehabil Med 37:698-705

61. Patrick DL, Burke LB, Gwaltney CJ, Leidy NK, Martin ML, Molsen E et al (2011) Content validity-establishing and reporting the evidence in newly developed patient-reported outcomes (PRO) instruments for medical product evaluation: ISPOR PRO good research practices task force report: part 1-eliciting concepts for a new PRO instrument. Value Health 14:967-977

62. Patrick DL, Burke LB, Gwaltney CJ, Leidy NK, Martin ML, Molsen E et al (2011) Content validity-establishing and reporting the evidence in newly developed patient-reported outcomes (PRO) instruments for medical product evaluation: ISPOR PRO Good Research Practices Task Force report: part 2-assessing respondent understanding. Value Health 14:978-988

63. Plinsinga ML, Coombes BK, Mellor R, Vicenzino B (2020) Individuals with persistent greater trochanteric pain syndrome exhibit impaired pain modulation, as well as poorer physical and psychological health, compared with pain-free individuals: a crosssectional study. Pain Med. https://doi.org/10.1093/pm/pnaa047

64. Prinsen CAC, Mokkink LB, Bouter LM, Alonso J, Patrick DL, de Vet HCW et al (2018) COSMIN guideline for systematic reviews of patient-reported outcome measures. Qual Life Res 27:1147-1157

65. Prinsen CAC, Vohra S, Rose MR, Boers M, Tugwell P, Clarke M et al (2016) How to select outcome measurement instruments for outcomes included in a "Core Outcome Set"-a practical guideline. Trials 17:449
66. R Core Team (2020) A language and environment for statistical computing. R Foundation for Statistical Computing. Vienna, Austria

67. Rabusin CL, Menz HB, McClelland JA, Evans AM, Landorf KB, Malliaras P et al (2019) Efficacy of heel lifts versus calf muscle eccentric exercise for mid-portion Achilles tendinopathy (the HEALTHY trial): study protocol for a randomised trial. J Foot Ankle Res 12:20

68. Rio E, Moseley L, Purdam C, Samiric T, Kidgell D, Pearce AJ et al (2014) The pain of tendinopathy: physiological or pathophysiological? Sports Med 44:9-23

69. Robinson JM, Cook JL, Purdam C, Visentini PJ, Ross J, Maffulli N et al (2001) The VISA-A questionnaire: a valid and reliable index of the clinical severity of Achilles tendinopathy. Br J Sports Med 35:335-341

70. Sierevelt I, van Sterkenburg M, Tol H, van Dalen B, van Dijk N, Haverkamp D (2018) Dutch version of the Victorian Institute of Sports Assessment-Achilles questionnaire for Achilles tendinopathy: reliability, validity and applicability to non-athletes. World J Orthop 9:1-6

71. Silbernagel KG, Thomee R, Karlsson J (2005) Cross-cultural adaptation of the VISA-A Questionnaire, an index of clinical severity for patients with Achilles tendinopathy, with reliability, validity and structure evaluations. BMC Musculoskelet Disord $6: 12$

72. Terwee CB, Bot SDM, de Boer MR, van der Windt DAWM, Knol DL, Dekker J et al (2007) Quality criteria were proposed for measurement properties of health status questionnaires. J Clin Epidemiol 60:34-42

73. Terwee CB, Jansma EP, Riphagen II, de Vet HC (2009) Development of a methodological PubMed search filter for finding studies on measurement properties of measurement instruments. Qual Life Res 18:1115-1123

74. Terwee CB, Prinsen CAC, Chiarotto A, Westerman MJ, Patrick DL, Alonso J et al (2018) COSMIN methodology for evaluating the content validity of patient-reported outcome measures: a Delphi study. Qual Life Res 27:1159-1170

75. Turner J, Malliaras P, Goulis J, Mc Auliffe S (2020) "It's disappointing and it's pretty frustrating, because it feels like it's something that will never go away." A qualitative study exploring individuals' beliefs and experiences of Achilles tendinopathy. PLoS ONE 15:e0233459-e0233459

76. Van der Doelen T, Jelley W (2020) Non-surgical treatment of patellar tendinopathy: a systematic review of randomized controlled trials. J Sci Med Sport 23:118-124

77. van der Vlist AC, Winters M, Weir A, Ardern CL, Welton NJ, Caldwell DM et al (2020) Which treatment is most effective for patients with Achilles tendinopathy? A living systematic review with network meta-analysis of 29 randomised controlled trials. Br J Sports Med. https://doi.org/10.1136/bjsports-2019-101872

78. Vicenzino B, de Vos RJ, Alfredson H, Bahr R, Cook JL, Coombes BK et al (2020) ICON 2019-International Scientific Tendinopathy Symposium Consensus: there are nine core health-related domains for tendinopathy (CORE DOMAINS): Delphi study of healthcare professionals and patients. Br J Sports Med 54:444-451

79. Viechtbauer W (2010) Conducting meta-analyses in R with the metafor package. J Stat Softw 36:1-48

80. Visentini PJ, Khan KM, Cook JL, Kiss ZS, Harcourt PR, Wark JD (1998) The VISA score: an index of severity of symptoms in patients with jumper's knee (patellar tendinosis). J Sci Med Sport $1: 22-28$

81. Wageck BB, De Noronha MA, Lopes AD, Da Cunha RA, Takahashi RH, Costa LOP (2013) Cross-cultural adaptation and measurement properties of the Brazilian Portuguese version of the Victorian Institute of Sport Assessment-Patella (VISA-P) scale. J Orthop Sports Phys 43:163-171 
82. Ziegler M, Hagemann D (2015) Testing the unidimensionality of items. Eur J Psychol Assess 31:231-237

83. Zwerver J, Kramer T, van den Akker-Scheek I (2009) Validity and reliability of the Dutch translation of the VISA-P questionnaire for patellar tendinopathy. BMC Musculoskelet Disord 10:102
Publisher's Note Springer Nature remains neutral with regard to jurisdictional claims in published maps and institutional affiliations.

\section{Authors and Affiliations}

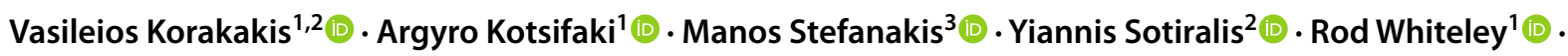 Kristian Thorborg ${ }^{4}$ (i)}

1 Aspetar, Orthopaedic and Sports Medicine Hospital, PO 29222, Doha, Qatar

2 Hellenic Orthopaedic Manipulative Therapy Diploma (HOMTD), Athens, Greece

3 School of Science, Program of Physiotherapy, University of Nicosia, Nicosia, Cyprus
4 Department of Orthopaedic Surgery, Sports Orthopedic Research Center-Copenhagen (SORC-C), Amager-Hvidovre Hospital, Faculty of Health Sciences, Copenhagen University, Copenhagen, Denmark 